\title{
Chronic Infection and Enterocutaneous Fistula Secondary to Mesh Migration and Erosion into the Small Bowel
}

\author{
Joao Baptista Rezende-Neto, Sarah Marshall, Kirsty Nixon, Paraskevi A. Vlachou, and Ori Rotstein
}

\begin{abstract}
Background: The use of prosthetic mesh is associated with a lower risk of hernia recurrence and hence is used commonly in hernia repair. Mesh migration and erosion into the gastrointestinal tract, albeit rare, is one of the most serious and challenging complications of hernia operations. Timely surgical intervention is an essential part in the management of this condition.

Case Presentation: A 55-year-old Caucasian female was admitted with severe abdominal pain and chronic abdominal wall infection nine years after an incisional hernia repair with mesh. Computed tomography (CT) scan of the abdomen revealed mesh eroded into the small bowel with a surrounding abscess and a fistulous tract. Surgery involved resection of the small bowel segment containing the mesh followed by primary anastomosis and complete excision of the remaining mesh. An adnexal pelvic mass was also excised. Despite a postoperative surgical site infection the patient was well at follow-up.

Conclusion: This report emphasizes the importance of timely surgical intervention in patients with mesh erosion into the gastrointestinal tract. Moreover, surgeons should be aware of the increased risks of mesh erosion and enterocutaneous fistula if native tissue is not interposed between the mesh and the bowel in underlay and sub-fascial hernia repair techniques.
\end{abstract}

Keywords: bowel; fistula; hernia; infection; mesh; perforation

$\mathbf{M}$ ORE THAN 100,000 incisional hernias are repaired every year in the United States [1,2]. Primary suture of incisional hernias results in recurrence rates as high as 50\% [3]; therefore, prosthetic mesh are routinely used in these operations. Major advancements in prosthetic materials have significantly reduced the recurrence rates to $10 \%-20 \%$ [4,5]. Nevertheless, several mesh-related complications have been reported posthernia repair [6,7]. These complications are associated with type of mesh, intra-operative findings, and the surgical technique. We report a case of mesh migration resulting in chronic infection and gastrointestinal fistula after incisional hernia repair.

\section{Case Presentation}

A 55-year-old Caucasian female was admitted to our hospital with severe abdominal pain and chronic abdominal wall infection. The patient had an extensive history of previous abdominal operations, including cholecystectomy, tubal ligation, hysterectomy, and an emergency laparotomy for perforated diverticulitis complicated by sepsis and a colovesical fistula. The last abdominal operation had been performed nine years prior to the admission described herein. The procedure was an underlay repair of an incisional hernia performed with a polypropylene and expanded polytetrafluoroethylene (ePTFE) bilayer mesh. The procedure was complicated by post-operative hematoma and site infection. In the following years, the patient developed chronic abdominal pain, episodes of lower gastrointestinal bleeding, bowel obstruction, and sporadic infection flare-up with purulent discharge from the site of the hernia repair.

At the present admission, physical examination revealed a distended abdomen with erythema, swelling, and tenderness on the umbilical region extending to the left lumbar region. There were faint bowel sounds; the patient was febrile (temperature, $38^{\circ} \mathrm{C}$ ) and hemodynamically stable. Laboratory tests showed leukocytosis of 19,020 per microliter $(15,690$ neutrophils per microliter), hemoglobin $10.5 \mathrm{~g} / \mathrm{dL}$, and a normal anion gap. Chest radiograph was normal.

St. Michael's Hospital-Division of General Surgery, Toronto, Ontario, Canada.

(C) Joao Baptista Rezende-Neto et al. 2017; Published by Mary Ann Liebert, Inc. This Open Access article is distributed under the terms of the Creative Commons License (http://creativecommons.org/licenses/by/4.0), which permits unrestricted use, distribution, and reproduction in any medium, provided the original work is properly credited. 
Computed tomography (CT) scan of the abdomen and pelvis showed a thick-walled fluid collection $(6.5 \times 1.9 \times 6.2 \mathrm{~cm})$ containing a folded mesh and locules of air in keeping with an abscess (Fig. 1). This collection was just posterior to the rectus abdominis muscles, extending from the level of the umbilicus to the apex of the urinary bladder. Furthermore, there was a fistulous tract extending from the caudal end of the abscess to the skin. A shriveled mesh was seen within the abscess. This mesh appeared to have eroded into the adjacent small bowel. A smaller abscess was seen anterior to the abdominal wall musculature in the left lower abdomen. This collection was connected to the one previously described and was also complicated by a fistulous tract extending to the skin. Pelvic images revealed a right adnexal cyst $(5.3 \times 4.4 \times 5.3 \mathrm{~cm})$. Intravenous antimicrobial therapy was initiated (cefazolin and metronidazole) and a midline laparotomy was performed. Surgical findings demonstrated extensive intraabdominal adhesions and a large segment of polypropylene mesh (approximately $20 \times 25 \mathrm{~cm}$ ) eroded partially into a loop of small bowel (Fig. 2). Furthermore, an abscess was seen encased between the other segments of bowel adjacent to the mesh and the perforation. The small bowel proximal to this area was significantly hypertrophied because of the distal obstruction. A segment of approximately $50 \mathrm{~cm}$ of small bowel containing the mesh involved in the perforation was resected followed by a primary side-to-side anastomosis. The distal small bowel was intimately adhered to the adnexal mass. Given the possibility of malignancy the mass was removed, the omentum was biopsied, and a pelvic peritoneal washing was performed for cytology. Subsequently, the remaining mesh was completely excised from the abdominal wall. The ensuing fascial defect was sutured with interrupted

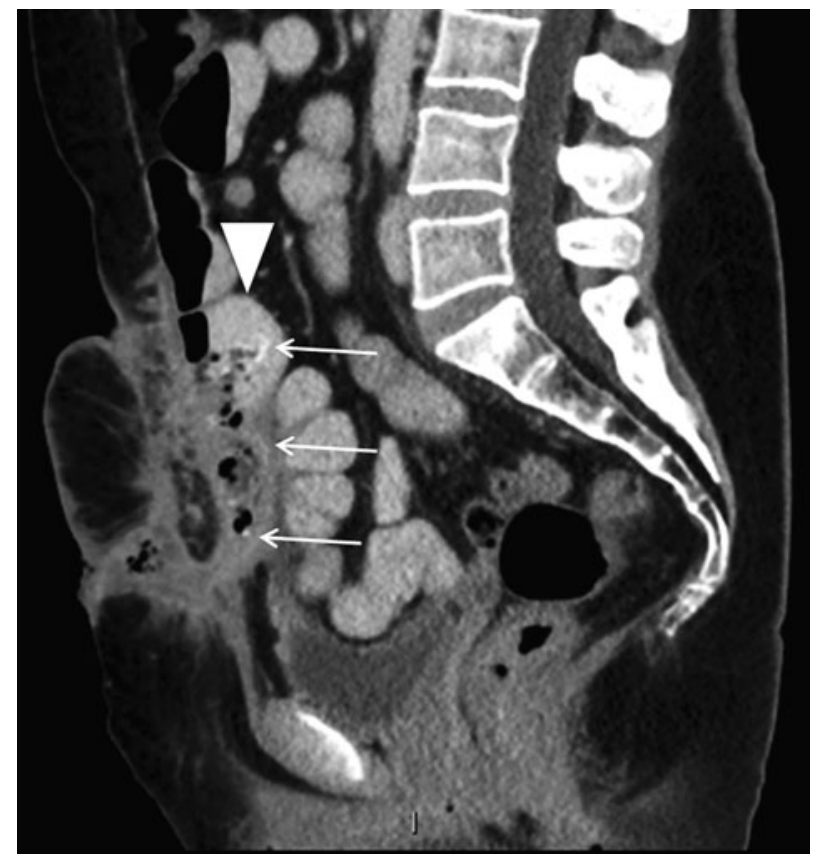

FIG. 1. Contrast enhanced computed tomography (CT) scan of the abdomen and pelvis, sagittal view, showing an abscess behind the anterior abdominal musculature with a fistulous tract extending to the skin surface at the inferior aspect. A shriveled mesh is seen within the abscess (arrows) eroded into an adjacent small bowel loop (arrowhead).

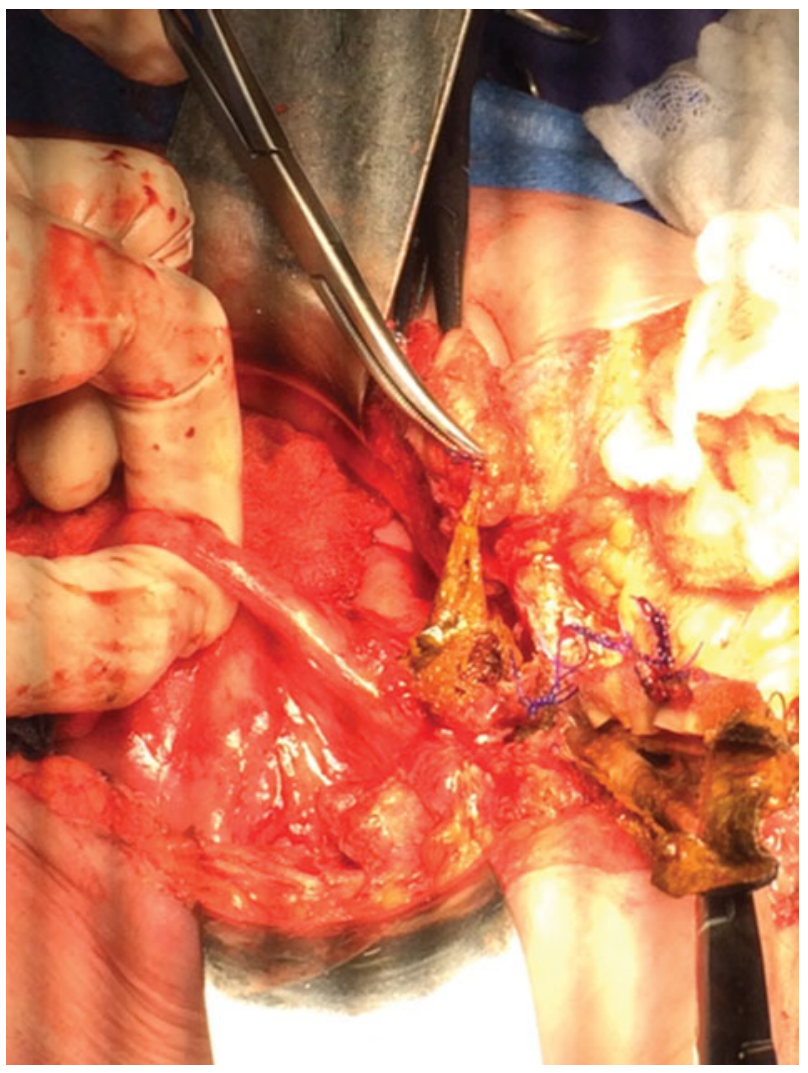

FIG. 2. Intra-operative photograph depicts mesh eroded into the small bowel.

stitches. The skin was closed over continuous suction drains placed in the subcutaneous tissue. The procedure was complicated by prolonged post-operative ileus and post-operative surgical site infection. The latter was treated with wound packing. The patient was discharged from the hospital on post-operative day 14

The histopathology report of the specimens disclosed areas of mucosal necrosis, acute inflammation with mucopurulent exudate, fibrotic lamina propria, and acute serositis of the small bowel. The adnexal mass was a benign cystadenoma and the omental biopsy was negative for malignancy. No complications were observed at the last follow-up visit five months after the operation.

\section{Discussion}

Mesh migration and erosion into the bowel is an unusual long-term problem after incisional hernia repair. A systematic review of late mesh-related complications in elective hernia repairs revealed only 69 long-term complications published over a 20-year span (1991-2011) [5]. In that review, there were 29 cases of mesh migration and 17 cases of fistula formation. Accounts of long-term mesh-related complications consistently describe a gradual process that develops over a period of more than one to three years and could present as late as 30 years after the repair of the hernia [4-6,8]. Therefore, the actual incidence of this problem remains uncertain and is possibly underreported [6-8]. Patients with mesh migration frequently relate a history of chronic abdominal pain and periods of intermittent bowel obstruction. Furthermore, 
chronic drainage of purulent matter through the skin overlying the repair with periods of infection flare-up are also common. Ultimately, mesh erosion into the bowel often results in gastrointestinal bleeding as described herein and enterocutaneous fistula. The mechanisms behind mesh migration and erosion are associated with poor fixation of the mesh to the surrounding tissue and foreign body reaction to the type of prosthetic material, particularly polyester fiber $[4,6]$.

Mesh migration is for the most part unpredictable. However, it is conceivable that underlay and sub-fascial techniques could potentially increase the chances of mesh erosion and enterocutaneous fistula if native tissue is not interposed between the mesh and the bowel [4]. Actually, excision of the hernia sac, presence of a fascial gap underneath the mesh, and lack of omental interposition are associated with a higher incidence of this complication [4]. Therefore, one should always prevent contact between intra-abdominal viscera and the mesh, even if the mesh contains a non-adhesive inner surface. Last, early wound complications such as seroma, hematoma, and unresolved infection could potentially result in late infectious complications after incisional hernia repair [5].

Surgery is the cornerstone of treatment for gastrointestinal fistula caused by mesh erosion. Although the operation is often performed on an elective basis, the presence of bowel obstruction and peritonitis call for emergency interventions $[1,7,9]$. A recent study showed that delayed repair (more than $24 \mathrm{~h}$ ) of obstructive ventral hernias was associated independently with worse outcomes [7]. After adjusting for comorbidities, the authors reported higher rates of concurrent bowel resection, surgical site infection, and a twofold increase in mortality in patients who underwent surgery more than $24 \mathrm{~h}$ after admission [7]. Unfortunately, specific data pertaining to recurrent incisional hernias previously repaired with mesh were unavailable in that study. However, similar results would be expected in that setting.

Even though intra-operative findings dictate the surgical strategy, mesh erosion invariably requires surgical resection of the affected bowel segment. Debridement followed by primary repair is normally performed if erosion occurs in other hollow viscera. Presence of infection and significant contamination generally dictate excision of the entire mesh, unless a biologic mesh was used [10]. Definitive repair of the ensuing fascial defect in the same operation is controversial. In the present case, we decided to perform primary suture repair of the defect given the high rate of local and systemic complications after emergency surgical treatment of incisional hernias. In support of this decision, a previous study reported that $15 \%$ of the patients who underwent emergency repair of incisional hernias died within 30 days [9]. Moreover, the authors also reported higher rates of post-operative complications, surgical site infection, and increased hospital length of stay compared with patients operated on an elective basis [9]. The use of biologic mesh could arguably facilitate definitive repair in the emergency setting. However, there is insufficient high-quality evidence for a comparative advantage of biologic mesh in the repair of complicated hernias, particularly when the mesh is used for bridging [10].

\section{Conclusion}

Mesh erosion into the gastrointestinal tract is a late complication of incisional hernia repair for which surgery is the mainstay of treatment. The surgical approach is dictated, for the most part, by local and systemic findings. Nevertheless, segmental bowel resection and mesh excision are fundamental elements of the surgical strategy.

\section{Author Disclosure Statement}

The authors declare no conflicts of interest. No funding was received for the purpose of this case report.

\section{References}

1. Beadles CA, Meagher AD, Charles AG. Trends in emergent hernia repair in the United States. JAMA Surg 2015; 150:194-200.

2. Poulose BK, Shelton J, Phillips S, et al. Epidemiology and cost of ventral hernia repair: Making a case for hernia research. Hernia 2012;16:179-183.

3. Luijendijk RW, Hop WCJ, van den Tol P, et al. Comparison of suture repair with mesh repair for incisional hernia. N Engl J Med 2000;343:392-398.

4. Leber GE, Garb JL, Alexander AI, et al. Long-term complications associated with prosthetic repair of incisional hernias. Arch Surg 1998;133:378-382.

5. Seker D, Kulacoglu H. Long-term complications of mesh repairs for abdominal-wall hernias. J Long Term Eff Med Implants 2011;21:205-218.

6. Gandhi D, Marcin S, Xin Z, et al. Chronic abdominal pain secondary to mesh erosion into cecum following incisional hernia repair: a case report and review of the literature. Ann Gastroenterol 2011;24:321-324.

7. Sippey M, Pender JR, Chapman WHH, et al. Delayed repair of obstructing ventral hernias is associated with higher mortality and morbidity. Am J Surg 2015;210:833-837.

8. Millas SG, Mesar T, Patel RJ. Chronic abdominal pain after ventral hernia due to mesh migration and erosion into the sigmoid colon from a distant site: A case report and review of literature. Hernia 2015;19:849-852.

9. Altom LK, Snyder CW, Gray SH, et al. Outcomes of emergent incisional hernia repair. Am Surg 2011;77:971-976.

10. Montgomery A. The battle between biological and synthetic meshes in ventral hernia repair. Hernia 2013;17:3-11.

Address correspondence to:

Dr. Joao Baptista Rezende-Neto

St. Michael's Hospital

Division of General Surgery

30 Bond Street, Room 3073

Toronto, Ontario, M5B 1 W8

Canada

E-mail: rezendenetoj@smh.ca

$\begin{aligned} & \text { Abbreviations Used } \\ \mathrm{CT}=\text { computed tomography } & \text { expanded polytetrafluoroethylene } \\ \mathrm{ePTFE} & =\text { expand }\end{aligned}$

Cite this article as: Rezende-Neto JB, Marshall S, Nixon K, Vlachou P, Rotstein O (2017) Chronic infection and enterocutaneous fistula secondary to mesh migration and erosion into the small bowel. Surgical Infections Case Reports 2:1, 17-19, DOI: 10.1089/crsi.2017.0003 